\title{
在 Python 语言程序设计中培养创新思维的研究
}

\section{Research of Cultivating Innovative Thinking in Python Programming 刘尚懿}

Shangyi Liu

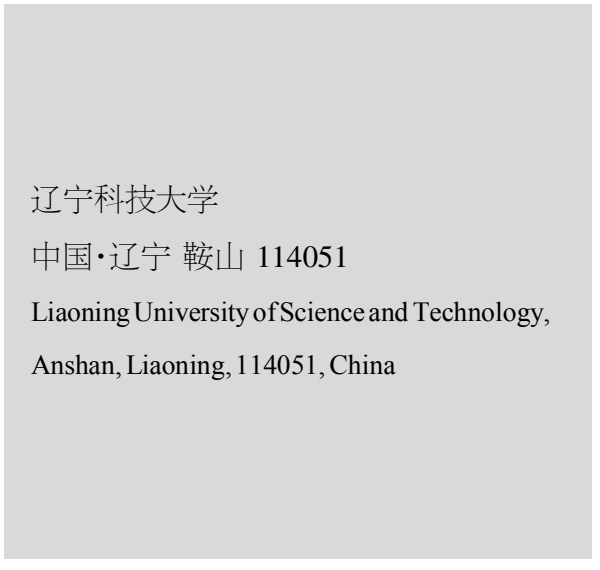

【摘要】论文分析了一些基本编程中常用的教学语言, 比较了 Python 和其他语言的功 能, 并讨论了在改革基本编程中使用 Python 的重要性以及在进行语言程序设计的过程中 如何培养学生的创新思维。

【Abstract】This paper analyzes some teaching languages commonly used in basic programming, compares the functions of Python and other languages, and discusses the importance of using Python in the reform of basic programming and how to cultivate students' innovative thinking in the process of language programming.

【关键词】语言程序;程序设计;创新思维

【Keywords \language program; program design; innovative thinking

【DOI】10.36012/sde.v2i1.778

\section{1 引言}

Python 语言已经发展了近 30 年, 其教育内容主要是基本 的语法、句子结构和函数库。Python 语言具有可以对故障排 除、语法宽松、功能强大的数据库, 并且可以编写执行复杂计 算的简单程序的优势。Python 具有接近自然语言的高级语 言表达能力, 对于专业和非专业学生, Python 是一种很好的编 程语言。Python 语言抽象了问题和解决方案, 具有程序重用 的概念, 可以满足大学编程语言基础课程的需求。

\section{Python 语言简介}

\subsection{Python 语言符合时代特征}

如今, 程序编写的过程中出现了大量的可用语言, 它们可 以反映计算思想, 但是却都有一些相应的缺陷。计算机编程的 基础课程应教授一种编程语言, 以便学生能够使用计算思维来 解决常见的计算问题。对于专业学生, 有必要继续加深这种能 力, 并在随后的课程中确定学习方向。非专业学生应将此功能 应用到他们的专业上, 以充分利用计算机的强大功能来解决 计算问题。因此,Python 作为入门语言是一个合理的选择 ${ }^{[1]}$

高级编程语言一直在寻求与人类接近的自然语言, 同时 避免了歧义并隐藏了计算机系统的设计细节。C 语言、Java 语 言和 VB 语言在高级语言开发中的出现具有上述特征, 但是 与 Python 相比, 其他的语言过分强调语法, 不利于培养计算 思维。Python 的教育价值体现在其轻量级的语法和功能上, 开
发更简洁的代码以实现更强大的功能, 帮助学生更快地入门 和更有效地开发, 以及更好地理解和实践计算机思维。在当今 的信息社会中, 对大学生编程技能的要求不断提高, 在这个时 代, 编程过程的教育内容也必须改变, 在进行教学的过程中必 须注重学生创新思维的培养。

\subsection{Python 语言功能}

Python 是一种开放源代码编程语言, 它使用一种弱类型 的脚本语言, 没有指针、地址和其他计算机系统结构元素, 在 使用它们之前无须进行声明, 它们具有独立的字符串类型, 并 且使用可变长度列表而不是固定长度数组, 列表与许多数据 类型兼容, 因此脚本语言直接通过解释器运行源代码。Python 语言的特点是代码相对简洁, 不仅支持面向过程的编程方法, 还支持面向对象的编程方法。

Python 是现代世界中常用的常见编程语言之一, 具有高 度的抽象性和高效的编码, 反映了计算思想的先进性, 可以解 决常见的计算问题并适应计算机级开发, Python 语言还可满 足计算机世界发展需求的开源系统。Python 语言的程序编写 系统公共社区提供了一个涵盖超过 68956 个功能的库, 涵盖 许多技术领域, 以及一个供 Python 语言作者学习、交流和发 展的平台。对于那些使用 Python 的人来说, 一些新兴的技术 领域已成为他们的良好就业前景。Java 语言主要是为 Android 系统开发的应用程序, 而 Python 语言具有更广泛的应用程 序, 包括数据分析师、运营和维护工程师以及产品测试。 


\section{3 在 Python 语言的教学中培养创新性思维}

国际大学发展中的一个问题是编程语言课程的教学内容 无法达到教学目的,美国大学引入 Python 语言进行了教学改 革, 并取得了显著成果。斯坦福大学于 2009 年开始提供 Python 课程, 以取代 Java 和某些 C 语言, 并获得了出色的教 学质量和成绩。中国大学在 Python 方面的培训实践相对落 后,必须进行教学模式的创新。

\section{1 设置合理的课程安排}

Python 语言教学课程共 64 个小时, 其中 40 个小时是理 论课程,每周 4 个小时,计算机实践 24 个小时,前 8 周每周 2 个 小时,接下来 2 周每周 4 个小时。学习 Python 基本语法的教 科书是北京工业大学宋天先生编写的《Python 语言程序设计 基础》第二版。对于学生来说, 在有限的时间内快速入门并了 解基本的语法知识非常重要, 而后续的学生将能够尽快使用 Python 解决实际问题并培养计算思维和创新技能更加关键。

\section{2 使用开放实验室项目}

为了配合这项教育改革, 以更好地培养学生的计算机思 维和创新意识,并解决由程序设计引起的现实问题,该课程从 10 周开始进行了 32 小时的开放实验室项目，综合实验专注 于每个教室的应用,学生学习了许多主题,开发了全面的小型 项目,并编写了项目实验报告。学生可以自主选择一个主题来 完成一个全面的开放项目,可充分反映学生的学习热情。学生 开始进行主动地主题选择、小组讨论、课程库安装和逐步的代 码调试, 以提高实际解决问题的能力, 激发学生的创造力。通过 对实验室开放的项目, 可以有效地增加学生的培养实践能力的 机会, 让学生能够有更多的机会利用 Python 语言进行实际问 题的解决, 提高学生的创新能力和思考能力。实验室开放项目 需要校方的支持, 为教学提供相应的教学场所和教学资源。

\section{3 教学方法和教育模式的改革}

上课前, 教师要求学生使用 MOOC 和微信公众号完成在 线预览, 在上课期间, 学生指导并启发学生在预览中思考问 题, 并讨论如何解决该问题。课后, 学生要求解决一些综合案 例。通过对整个课程的全新设计, 可以有效地提高课程的有效 性, 让教师在教学的过程中更加有效率, 让学生在学习的过程 中取得更多的成果。根据“Python 编程”课程内容的 3 个级别, 建议具体的学习任务, 设计一系列微型视频(如 MOOC), 设置 作业前项目任务, 让学生在上课前可以提前预习课程相关的 要点和内容。根据课程课程的设计需求, 教师使用 $Q Q$ 群组和 微信向学生介绍作业和要求, 学生可以在学习网站上准备目 标课程, 并根据其基础知识和时间下载并保存学习资源。在老
师的指导下, 学生们根据自己的倡议组织研究小组, 以便在小 组负责人的带领下尽早完成项目小组、程序配置、项目测试和 课前实验检测任务。

上课过程中, 教师需要明确教学目标, 教学目标是指导学 生解决自我学习的理论和常见问题, 同时指导学生消化理论 知识并应用和扩展理论知识,包括项目任务复习、问题解决和 学生自学。课程过程中教师需要做好记录, 统一并反馈项目实 施中出现的问题和常见问题,评估项目的完成情况, 并鼓励学 生在课堂上交流和讨论创新思想。

下课后, 在完成“Python 编程”课程自学项目后, 老师将及 时为综合应用添加模块化测试, 以使学生能够总结和发现缺 失的领域。Python 编程课程采用综合评估,强调学生的专业素 养和专业技能的发展, 测试学生的 Python 程序设计技能并提 高学生的整体业务水平。“Python 编程”课程按组完成项目工 作, 每个组有 2 3 个成员, 最终分数包括综合质量评估 (20\%), 过程评估 $(30 \%)$ 和最终评估 $(50 \%)$, 最终评估包括工作选择、 任务完成、工作态度和创新技能。学生必须展示和解释作业并 回答老师提出的问题,最后,每个学生组均需提交完整的课程 设计报告和操作程序。

\section{4 教育改革成就}

基于结果的教学概念基于实际需求, 并进行反向设计课 程安排, 同时提高学生对知识的掌握能力, 因此, 学生可以练 习和提高他们的编程技能。在“Python 编程”课程的教学改革 的早期实施中,在宏观层面采用了基于项目的教育, 在微观层 面采用了基于问题的教育,扩展作业中需要解决的问题,并使 用前后知识点之间的相关性来指导学生相互学习, 学生可以 应用的知识内容也得到了提高。在进行教学改革的过程中, 已 经取得了一些成就, 提高了学生的实践能力和创新能力, 但是 要想培养学生独立解决实际问题的能力还需要进一步进行教 学方法的改善, 培养学生的学习兴趣。

\section{4 结语}

对于没有计算机编程基础的学生, 可以通过学习 Python 语言提高自己的综合素质,扩宽就业前景, 要想在进行 Python 语言的教学过程中培养学生的创新思维, 必须对教学的时间 和形式安排进行改革。同时,研究组的教师必须不断优化教学 内容和教学方法, 利用多样化的信息技术提高教育效率和质 量, 实现培养具有解决创新意识、计算思维和实际问题能力的 学生的教育目标。

\section{参考文献}

[1]曹洁,崔霄.面向新工科的 Python 数据分析课程内容浅析 [J]. 河南教育(高教),2019(7):95-97. 